AperTO - Archivio Istituzionale Open Access dell'Università di Torino

\title{
Abduction in Machine Learning
}

\section{This is the author's manuscript}

Original Citation:

Availability:

This version is available http://hdl.handle.net/2318/122311

since

Publisher:

Kluwer Academic Publishers

Terms of use:

Open Access

Anyone can freely access the full text of works made available as "Open Access". Works made available under a Creative Commons license can be used according to the terms and conditions of said license. Use of all other works requires consent of the right holder (author or publisher) if not exempted from copyright protection by the applicable law. 
[Tarski, 1936] A. Tarski. Über den Begriff der logischen Folgering, Actes du Congrès Int. de Philosophie Scientifique 7:1-11, 1936. Translated into English as On the concept of logical consequence. [Zadrozny, 1991] W. Zadrozny. On rules of abduction. IBM Research Report, August 1991.

\section{ABDUCTION IN MACHINE LEARNING}

\author{
1 INTRODUCTION
}

Both inductive learning and abductive reasoning start from specific facts or observations and produce some explanation of these facts. Both may be described as forms of defeasible reasoning from effects to causes. There are some differences, but they are minor and due to different understandings of the notions of observation and explanation (see for instance [Bergadano and Besnark, 1994]). We build on the general notions developed in the introductory Chapter, taking what was labeled there as the syllogistic view, in the sense that we isolate the differences between abduction and induction based on syntactic considerations.

bduction and induction based on syntactic considerations.

Briefly, induction sees examples as instances of a concept and explanations as
general concept descriptions, whereas abduction sees examples as specific observations and explanations as other specific facts that are true, and cause the observations to occur. As a typical case for induction, an example could be the description of a specific bird, and a concept description could be a rule such as

$$
\operatorname{bird}(X) \leftarrow \operatorname{small}(X) \wedge \operatorname{part} \_o f(X, Y) \wedge \operatorname{wing}(Y)
$$

with the obvious intended meaning. For abduction, an observation could be the fact that some particular bird does not fly, and an explanation could be the fact that that particular bird has a broken wing.

In both cases, the relevant logical relation is that the explanation, together with the domain knowledge, implies the observation. This aspect is developed in depth in the introductory Chapter.

The Machine Learning literature has largely ignored such similarities, or has produced studies that emphasize the differences that are present [Console and Saitta, 1993; Flach, 1992]. On the other hand, abduction has been used as an effective technique within the underlying inductive framework of Machine Leameffective tech ique within ing methods. Two different approaches, which we think are quite represen
the Machine Learning view of abductive reasoning, can be identified.

- First, abduction has been used to guide search in top-down relational learning. The idea has evolved from explanation-based learning methods, restricting the inductive hypotheses to be logical consequences of a given domain theory. This is then generalized by allowing inductive hypotheses to be obtained from the domain theory by either deductive or abductive reasoning.

- Second, abduction has been used to generate missing examples in relationa learning. In fact, it is intrinsic to the nature of induction that the input data is incomplete: some examples are given, but not all. If some particular 197 
examples are missing, existing relational learning methods may however encounter serious problems, and abductive generation of these missing examples may be an effective solution.

Top-down relational learning algorithms suffer from the difficulty of searching a space of possible inductive hypotheses that is usually very large. In the first relation [Quin, 1990], he problem was addesed with heuristics of a statistical na[Quinlan, 190], the problem was addresed with heuristics of a statical nature. Top-down systems start from very general concept descriptions, and then try to obtain consistent rules with a number of specialization steps. Typically, heuristics would favor specializations that exclude negative examples while still covering a large number of positive examples. Such heuristics may be misleading and may also be insufficient for an adequate pruning of the hypothesis space. The Explanation-Based Learning (EBL) paradigm restricts the concept descripThe Explat the logical consequences of a so-called thons that may be possibly produced to he logical con domain theory, that is given as input to the learning system IMitchell et al., 1986; Bergadano and Giordana, 1988; Pazzani and Kibler, 1992]. Abduction has been used in this framework as follows: given the domain theory, the concept descriptions that may be obtained via abductive reasoning are considered as possibly true, and should be evaluated inductively on the basis of the available examples. Studies that follow this scheme may be found in [Bergadano et al., 1989; Cohen, 1992 Cohen, 1994].

A well known problem in Machine Learning is to provide a learning method with an "adequate" set of examples of the target concept. Here "adequate" informally means that the training set should contain all those examples required to successfully complete the learning task, and no more. Obviously, this is a very hard condition to achieve, since usually it is not possible to know in advance exactly which examples are (and which are not) significant for learning a conce to slow (if concept. As a cols too many examples are given) andor may fall (if the exanples are not significant). This problem is particularly serious for an important class of learning methods: Relational Learning Algorithms based on an extensional interpretatio of sub-predicates and recursion [Quinlan, 1990; Bergadano and Giordana, 1988; Pazzani and Kibler, 1992]. In these methods, the learning procedure can not only fail or be too slow, but also produce wrong results: the description of the target concept synthesized by the system may entail some of the negative examples get concept synthesized
given to the system.

given to the system.
It is our objective to show how abduction can be used to fix the above problem. An abductive procedure is used to query the user for any example that may be missing, depending on the hypothesis space that has been defined and the given examples. A similar technique has been used before, for example in [DeRaedt and Bruynooghe 1991: Shapiro, 1983], to query the user for missing values allowing a Bingle correct and sufficient, in the sense that the learned description does not entail any of the negative examples and such a description can always be found if it exists. We also show how this technique can be adapted to the problem of program testing: a combination of induction and abduction can be used to generate an "adequate"
test set for a program under testing.

\section{ABDUCTION AND INDUCTION}

Abductive and Inductive Reasoning in Artificial Intelligence are considered to be distinct and have generated separate fields of study. After a simple analysis, one finds in effect distinct inference schemes.

$$
\begin{aligned}
& \text { - For induction: } \frac{P(a)}{\forall x P(x)} \\
& \text { - For abduction: } \frac{P(x) \rightarrow Q(x) \quad Q(a)}{P(a)}
\end{aligned}
$$

As stated in the introductory Chapter, a deeper analysis, however, suggests that the difference between the two schemes is not always easy to state. For instance, using the tautology

one gets:

$$
\Phi \equiv(\forall x P(x)) \rightarrow P(a)
$$

$$
\Phi \quad P(a)
$$

as an abductive inference step, but it actually has the same premises and conclusions of the inductive inference scheme.

It would then seem that the abductive scheme includes simple forms of inductive reasoning. In [Bergadano and Besnark, 1994] the authors start from the above considerations and then isolate some minor differences of abductive and inductive reasoning within the same framework, that is determined by the above inference rules. In the same paper, a formalization based on non-monotonic logic is develuped. and abduction are indeed very similar inference schemes. A deeper analysis is found in the introductory Chapter of the present Book, where general similarities are noted, and syntactic, inferential and semantic differences are considered.

However, the Machine Learning literature has not used abduction and induction as synonyms. The main keyword in learning is induction, and abductive reasoning is rather used as an additional technique for solving particular problems. We discuss in the following sections two of such uses of abduction in Machine Learning. In particular in the next section we survey its use as a form of bias for guiding the search in a top-down specialization. Then, we show how abductive reasoning and queries can solve some relevant problems in relational top-down learning, of both binary and fuzzy predicates. 


\section{GUIDING SEARCH WITH ABDUCTION}

One use of abduction in Machine Learning is related to the problem of guiding search in a top-down specialization. As the number of concept descriptions that may possibly be generated is, in general, very large, and since even the number of ins the examples can be large, learning systems need extra-evidential criteria to prune the search space. Deductive inference with a domain theory has been used to this purpose in ML-SMART [Bergadano and Giordana, 1988] and FOCL [Pazzani and Kibler, 1992]. Similarly, a domain theory could be used abductively within the same framework as in [Bergadano et al., 1989] and in [Cohen, 1992; Cohen, 1994].

Before we can suitably describe the details of abductive inference to prune the space of possible concept descriptions, we need to define a type of analytic learning called Explanation-based Learning (EBL), which has received much attention during the late eighties. A survey of EBL is found in [Ellman, 1989].

EBL needs as input one or more positive examples, as well as a so-called domain theory, which includes relevant prior knowledge. A resolution proof of the positive examples is produced, and the leaves of the proof tree are generalized and taken as the antecedent of a new rule for the target concept. Suppose, for instance, that the domain theory is the following

$$
\begin{aligned}
& C(X):-B(X), A(X, Y) . \\
& B(X):-R(X, Y) .
\end{aligned}
$$

$B(b)$.

$A(c, a)$.

$R(c, d)$

and a positive example for $C$ is $c$. It is then easy to see that $C(c)$ can be obtained deductively from the domain theory, where the leaves in the proof tree are $R(c, d)$ and $A(c, a)$. This produces the learned description for $\mathrm{C}$

$C(X):-R(X, Y), A(X, Z)$

The learned description is actually a logical consequence of the given domain theory, and could be obtained from the domain theory even without looking at the examples, by resolving the first two clauses in the domain theory. The role of the example is that of suggesting some consequences of the domain theory rather than others: the ones that are useful to deduce the positive example are chosen.

One natural question arises: what is the purpose of this form of learning, if its output is just a logical consequence of something that was already known? The answer is that, athough nothing really new is learned, the new form of knowledge may be more operatonal, that is to say either easer to use or leading to more efficient computations. In the above case, the positive example $c$, that is to say $C(c)=$ true may be deduced more efficiently from the learned clause than by the original domain theory, as one resolution step is saved. EBL may then be seen as a form of pre-compilation, or partial evaluation. The effectiveness of this form of learning speed-up depends on whether the given positive examples are representative of future cases: if this is not true, the learned clause may turn out to be useless, as the original domain theory would be necessary every time. Learned clauses that are useless in this sense will only take away some memory space without giving any computational advantage.

For this reason, systems soon started using EBL with many positive examples, so that only clauses that frequently proved to be useful would be kept. Other systems (e.g., [Bergadano and Giordana, 1988]) also introduced the possibility of using negative examples, in connection with the acceptance of a domain theory that might be partly incorrect. In this case, even clauses that follow deductively from the domain theory may be incorrect, and may cover negative examples. Clause way, the domain theory tions that are logical consequences of the domain theory are descriptions that may potentially be learned. The description which is actually produced would also be required to perform well with respect to the data, i.e. cover many positive examples and few negative examples. EBL may then be considered as a way to guide search in top-down specialization: not all specializations are possible, but only the ones that produce deductive consequences of the domain theory. Legal specializaions are obtained by resolving the clause to be speciaize tions are oblained by resolving the clause to be specialized aganst some clause in the domain theory. Other specializations are not considered. In the previou example, the clause

$$
C(X):-R(X, Y), A(X, Z)
$$

would be a legal specialization of the clause

$C(X):-B(X), A(X, Y)$

while, e.g., the clause

$$
C(X):-B(X), A(X, Y), B(Y)
$$

would not be considered. If the space of possible clauses is too large, a domain theory can be used effectively to prune such a space and suggest preferred specialization steps. ML-SMART [Bergadano and Giordana, 1988], and FOCL [Pazzani and Kibler, 1992] adopt similar goals and techniques.

Abduction comes into place if the consequences of the domain theory are not just taken to be deductive consequences, but are also obtained by means of a Just taken to be deducive conequences, th the be deductive con prequence, but are also ob in the above domain theory would contain the clause

$D(X):-A(X, Y)$

then, an abductive/deductive consequence of the theory would be the clause $C(X):-R(X, Y), D(X)$ 
which would also be a legal specialization of the clause

$$
C(X):-B(X), A(X, Y) .
$$

Such an abductive use of a domain theory is found in [Bergadano et al., 1989], with the purpose of restricting the hypothesis space without excluding concept descriptions that may be meaningful on the basis of abductive reasoning. In fact, such a use of abduction is just a means for specifying in a declarative way a form of inductive bias, a criterion for preferring an inductive hypothesis over another, or a rule for eliminating some description from the space of possible inductive generalizations. As suribs not see why a deductive apps a criterion is heuristic in nature, one would The deductive approach is the onould be more justified than an abductive one. to the integration of inductive reasoning in ML-SMART and other approache lows exactly the same scheme reasoning and EBL. The abductive approach fol$a l ., 1989$ ]. In this case the rule "as described, for example in [Bergadano $e$ sequence of a given dome rule "accept generalizations that are a logical conmay be ob a given domain theory" is replaced by "accept generalizations that me domain theory via abductive reasoning". In both that the obtained generalizations must in primis be consistent and . In both case spect to the given examples, or satisfy such requirement to a degree. The retion of abductive $E B L$ in a similar framework is introduced in [Co. The noCohen, 1994]

\section{RELATIONAL LEARNING ALGORITHMS BASED ON} EXTENSIONALITY

Relational Learning Algorithms learn recursive relational descriptions from positive and negative examples, given as ground literals. Usually, a subset of the firstorder predicate calculus, Horn clauses, is used for the concept description (see, e.g. [Birnbaum and Collins, 1991; Rouveirol, 1992; Cohen, 1992; Bergadano and Gunetti, 1993; Muggleton and Feng, 19901). Learning definite clauses for multiple predicates is difficult, and systems tend to be slow. Many systems, such as Foil
[Quinlan, 1990],

Focl [Pazzani and Kibler, 1992] and Golem [Muggleton and Feng, 1990] handle this problem by evaluating clauses extensionally. In this way candidate clauses can be generated directly from the examples one at a time and independently of one
another.

Let us now introduce the concept of extensionally defined predicate, which, intuitively, will play the role of the basis case in the recursive definition, which, complex predicates.

DEFINITION 1. A predicate $P(X)$ is said to be extensionally defined if it is ments $a_{1}, \ldots, a_{n}$, that is to say, if its
Examples of extensionally defined predicates are given by "mother" and "father" defined by the following table:

\section{Parenthood Table}

\begin{tabular}{|c|c|c|c|c|}
\hline \multicolumn{2}{|c|}{ mother } & \multicolumn{3}{|c|}{ father } \\
\hline $\mathrm{X}$ & $\mathrm{Y}$ & & $\mathrm{X}$ & $\mathrm{Y}$ \\
$\mathrm{d}$ & $\mathrm{g}$ & & $\mathrm{f}$ & $\mathrm{g}$ \\
$\mathrm{r}$ & $\mathrm{d}$ & & $\mathrm{s}$ & $\mathrm{d}$ \\
$\mathrm{r}$ & $\mathrm{p}$ & & $\mathrm{S}$ & $\mathrm{p}$ \\
$\mathrm{i}$ & $\mathrm{r}$ & & $\mathrm{b}$ & $\mathrm{r}$ \\
$\mathrm{t}$ & $\mathrm{s}$ & & $\mathrm{h}$ & $\mathrm{c}$ \\
$\mathrm{a}$ & $\mathrm{c}$ & & & \\
\hline
\end{tabular}

Some other predicates can be intensionally defined over a collection of extenionally defined predicates by means of simple logical rules.

An example is given by the predicate parent:

$$
\begin{array}{lll}
\operatorname{parent}(X, Y) & :-\operatorname{mother}(X, Y) \\
\operatorname{parent}(X, Y) & :- & \text { father }(X, Y)
\end{array}
$$

In this case, the extensional definition of the predicates mother and father provides as well an extensional definition of the predicate parent.

We formalize now the meaning of "extensional evaluation".

DEFINITION 2. Let $\alpha\left(X_{1}, X_{2}, \ldots, X_{n}\right)$ be a conjunction of predicates where there is at least one occurrence of each of the variables (not necessarily distinct) $X_{1}, \ldots, X_{n}$. We say that $\alpha\left(a_{1}, a_{2}, \ldots, a_{n}\right)$ is extensionally true if and only if

- in case $\alpha\left(X_{1}, \ldots, X_{n}\right) \equiv Q\left(X_{1}, \ldots, X_{n}\right)$, for some extensionally defined predicate $Q$, then $Q\left(a_{1}, \ldots, a_{n}\right)$ is a given positive example of $Q$

- in case

$$
\alpha \equiv \gamma\left(X_{i_{1}}, \ldots, X_{i_{h}}, T_{1}, \ldots, T_{l}\right), Q\left(T_{1}, \ldots, T_{l}, X_{j_{1}}, \ldots, X_{j_{k}}\right),
$$

for some conjunction $\gamma$, predicate $Q, l \geq 0$ and

$$
\left\{X_{i_{1}}, \ldots, X_{i_{h}}, X_{j_{1}}, \ldots, X_{j_{k}}\right\}=\left\{X_{1}, \ldots, X_{n}\right\}
$$

then there must exist $e_{1}, \ldots, e_{l}$ such that both the conjunction $\gamma\left(a_{i_{1}}, \ldots, a_{i_{n}}\right.$ $\left.e_{1}, \ldots, e_{l}\right)$ and $Q\left(e_{1}, \ldots, e_{l}, a_{j_{1}}, \ldots, a_{j_{k}}\right)$ must be extensionally true.

We say that the conjunct $\alpha\left(X_{1}, \ldots, X_{n}\right)$ extensionally covers a positive [resp. negative] example of $P, P\left(a_{1}, \ldots, a_{n}\right)$ [resp. $\neg P\left(a_{1}, \ldots, a_{n}\right)$ ] if and only if $\alpha\left(a_{1}, \ldots, a_{n}\right)$ is extensionally true.

The next subsection contains a simplified version of Foil, in order to illustrate the extensional approach and its drawbacks. 
4.1 The Extensional Approach: Simplified Foil

Let $P$ be the target concept and pos_examples $(P)$ and neg_examples $(P)$ the given positive and negative examples of $P$ (in the following, $\alpha$ and $\gamma$ represent generic conjunction of literals).

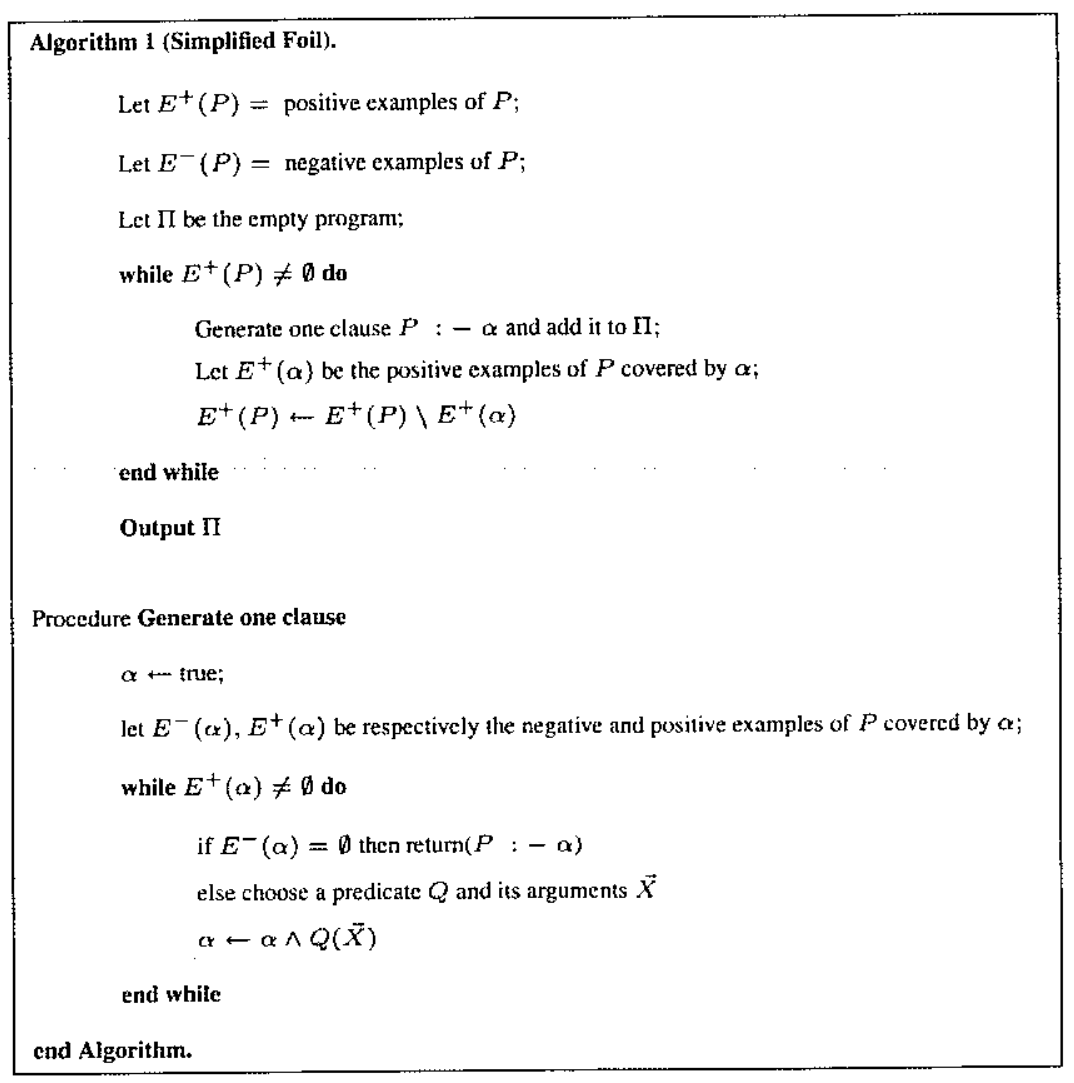

Every predicate $Q$ can be defined by the user either intensionally by or exten sionally. In particular, clauses can be recursive and, in this case, $Q=P$, and its truth value can only be determined by the available examples.

The choice of the literal $Q(\vec{X})$ to be added to the partial antecedent $\alpha$ of the clause being generated is guided by heuristic information. It might nevertheless be a wrong choice in some cases, in the sense that it may cause the procedure "Generate one claus" to fal by exiting the while loop without returning any clause. Thi problem can be fixed by making the choice of $Q(\vec{X})$ a backtracking point.

Suppose, for instance, that Foil is given the following positive and negative examples of the relation ancestor, which is the one to be learned:

$$
+\langle r, g\rangle,\langle b, g\rangle,\langle b, d\rangle,\langle d, g\rangle,\langle b, r\rangle,\langle r, d\rangle,\langle r, p\rangle
$$$$
-\langle d, d\rangle,\langle g, p\rangle,\langle d, p\rangle \text {. }
$$

Let us suppose as well that we have the intensional definition for the relation par ent given above along with the Parenthood Table which extensionally defines the ent given above along with the Parenthood Table which extensionally defines the
predicates mother and father. Finally, we know that the logic program for ancestor predicates mother and father. Finally, we know that the logic program for ancestor
depends on parent and on itself (i.e. it may be recursive). As there are at most 3 variables to be used, these are the possible literals:

parent $(X, Y)$, parent $(Y, X)$, $\operatorname{parent}(X, W)$,

parent $(W, X), \operatorname{parent}(Y, W), \operatorname{parent}(W, Y)$

ancestor $(X, W)$, ancestor $(W, X)$, ancestor $(Y, W)$, ancestor $(W, Y)^{1}$

The learning algorithm starts to generate the first clause - the antecedent $\alpha$ is initially equal to "true". We need to choose the first literal $Q(\vec{X})$ to be added to $\alpha$ As we have left the heuristics unspecified, we will choose it so as to make the discussion short.

Let $\alpha=$ parent $(W, Y)$; then all positive examples and the second and third negative examples are covered, so more literals need to be added.

Let $\alpha=\operatorname{parent}(W, Y) \wedge \operatorname{parent}(W, X)$; in this case no positive examples are covered and the negative example $<d, p>$ is covered. Clause generation fails and we backtrack to the last literal choice.

Let $\alpha=\operatorname{parent}(W, Y) \wedge$ ancestor $(X, W)$; no negative example is covered, and the first 3 positive examples are extensionally covered. A clause is generated and the covered positive examples are deleted.

We proceed to the generation of another clause; $\alpha$ is empty again. If we choose the first literal as parent $(X, Y)$, the remaining positive examples are covered and the final solution is obtained:

$\operatorname{ancestor}(X, Y):-\quad \operatorname{parent}(W, Y), \operatorname{ancestor}(X, W)$

ancestor $(X, Y):-\operatorname{parent}(X, Y)$.

Notice that the obtained definition of the relation "ancestor" is complete and consistent when completeness and consistency are characterized as follows.

DEFINITION 3. A definition $\Pi$ of $P$ is complete with respect to the positive examples $E^{+}$of $P$ if and only if

$$
\left(\forall e \in E^{+}\right) \Pi \vdash P(e) .
$$

A definition $\Pi$ of $P$ is consistent with respect to the negative examples $E^{-}$if and only if

$$
\left(\forall e \in E^{-}\right) \Pi \nvdash P(e) .
$$

$\mathrm{I}_{\text {ancestor }}(X, Y)$ and ancestor $(Y, \bar{X})$ are not listed because they may produce looping recursions 


\subsection{Problems of extensionality}

The independence of the clauses in the generation of the definition of "ancestor" is given by the extensional interpretation of recursion and sub-predicates: when a predicate $Q$ occurs in a clause antecedent $\alpha$, it is evaluated as true when the a predicate $Q$ occurs in a clause antecedent $\alpha$, it is evaluated as true
arguments match one of the positive examples. For instance, the clause

$$
\text { ancestor }(X, Y):-\operatorname{parent}(W, Y) \text {, ancestor }(X, W) .
$$

was found to extensionally cover the positive example $\langle b, g\rangle$ of ancestor because parent $(d, g)$ is true (see Parenthood Table) and $\langle b, d\rangle$ is also a positive example of ancestor. The previously generated logical definitions of $Q$ are not used. The method is (partially) justified by the following result (see [Bergadano used. The method is (

THEOREM 4. Suppose Foil successfully exits its main loop and outputs a logic program $\Pi$, that always terminates for the given examples.

Let $Q(X):-\alpha$ be a generated clause of $\Pi$, then, for any e positive example of $Q$, if $\alpha$ extensionally covers $e$ then $\Pi \vdash Q(e)$.

However, extensionality forces us to include many examples, which would otherwise be unnecessary. In fact other desirable properties, similar to the one given by Theorem 4, are not true, and two fundamental problems arise:

PROBLEM 5. For a logic program $\Pi$, it may happen that $\Pi \vdash Q(e)$, but none of its clauses extensionally cover $e$. As a consequence Foil would be unable to generate $\Pi$.

Consider for instance the program $\Pi$ :

$$
\text { ancestor }(X, Y):-\operatorname{parent}(W, Y) \text {, ancestor }(X, W) \text {. }
$$$$
\text { ancestor }(X, Y):-\operatorname{parent}(X, Y) \text {. }
$$

Let $\langle r, g\rangle$ be the only positive example of ancestor. As it is easy to see, by using resolution on $\Pi$ and the Parenthood Table this example logically follows from $\Pi$ (I $\vdash$ ancestor $(r, g)$ ). However, is not extensionally covered. Indeed, by inspecting the Parenthood Table, we can see that the second clause does not cover it because part $(r, g)$ is false and the first clause does not cover it extensionally example of ancestor.

PROBLEM 6. Foil may generate definitions which are not consistent. In details, even if no clause of a definition $\Pi$ extensionally covers a negative example $e$ of $Q$, it may still happen that $\Pi \vdash Q(e)$.

Consider for instance the following definition II for "ancestor":
$\operatorname{ancestor}(X, Y):-\operatorname{parent}(W, X), \operatorname{ancestor}(W, Y)$

ancestor $(X, Y):-\operatorname{parent}(X, Y)$.

It follows that $\Pi \vdash$ ancestor $(g, p)$. Nevertheless, $\langle g, p\rangle$ is not extensionally covered by the first clause: parent $(d, g)$ is true, but $\langle d, p\rangle$ is not a positive example of ancestor.

Since $d$ is not an ancestor of $p$, it could not possibly be added as a positive example, and $\langle g, p\rangle$ would not be extensionally covered even if all positive examples were given.

The solution for this problem differs from the one of problem 1: in this case II The solution for this problem differs from the one of problem 1 .
will be ruled out by adding a negative example, namely $\langle d, p\rangle$.

\section{PROBLEMS OF INTENSIONAL METHODS}

Giving up the extensional interpretation of predicates while keeping the basic computational structure of Foil produces unsolvable problems. In fact, the truth values of the missing examples could be obtained by means of the partial program generthed at a nen moment. But if the inductive predicates occurring in a clause being the order in which the clauses are learned becomes a major issue.

the order in which the clauses are learned becomes a major issue.
Suppose, for instance, that we are given as family tree the following subset of Suppose, for instance,
the Parenthood Table:

$\operatorname{parent}(i, r), \operatorname{parent}(r, d), \operatorname{parent}(d, g), \operatorname{parent}(f, g)$

as well as the following positive and negative examples of ancestor

$$
\begin{aligned}
& +\quad\langle i, d\rangle,\langle i, r\rangle,\langle i, g\rangle ; \\
& -\quad\langle i, f>.
\end{aligned}
$$

Suppose also that the following clauses are generated, with the given order:

(1) ancestor $(X, Y):-\operatorname{parent}(X, W)$, parent $(W, Y)$.

(2) $\operatorname{ancestor}(X, Y):-\operatorname{parent}(Y, W)$, ancestor $(X, W)$.

(3) $\operatorname{ancestor}(X, Y):-\operatorname{parent}(X, W)$, ancestor $(W, Y)$.

Clause (1) does not use any inductive predicate and immediately covers the positive tuple $\langle i, d\rangle$. Clause (2) contains ancestor $(X, W)$, and this literal is posaluated with the clauses available at this stage, i.e. (1) and (2). With this kind of intensional interpretation, the only example covered is $\langle i, r\rangle$, a positive example. When clause (3) is generated, it will cover the positive example $\langle i, g\rangle$. 
But clause (2) will now cover the negative example $\langle i, f\rangle$, since parent $(f, g)$ is true, and ancestor $(i, g)$ may now be deduced by using the third clause.

Rules that seem consistent and useful at some stage may later be found to cover negative examples. We must then backtrack to the generation of the clause causing the inconsistency, e.g. clause (3), and to the generation of the clause that was later found to be inconsistent, e.g. clause (2). In general, we must give up ou former assumption that clauses may be learned one at a time and independently. Alternatively, we may number the predicates occurring in the learned clauses every time an inconsistency is detected. For instance, the former program would be rewritten as

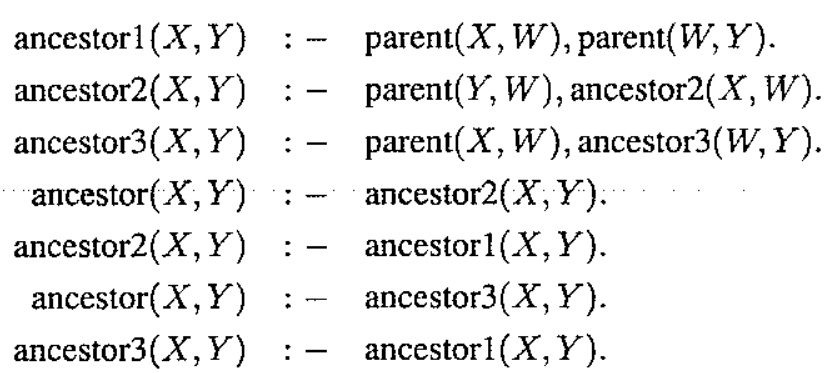

Nevertheless, this technique does not totally avoid the need for backtracking and explodes the number of possible clauses by multiplying the number of inductive predicates by the number of generated indexes. A solution based on abduction is presented in the next section, so that the advantages of extensionality are preserved, ie so that previously generated clauses do not need to be reconsidered.

\section{COMPLETING EXAMPLES VIA ABDUCTION BEFORE LEARNING}

There is no reason why particular positive (problem 1) or negative (problem 2) examples should have to be present. After all, the whole motivation of induction is that some information is missing. The important points are that

(1) if a definition $\Pi$ consistent with the given examples exists, then it must be found, and

(2) the induced definition II must not cover any negative examples.

The extensional approach guarantees neither, unless some specially determined positive and negative examples are given.

Here we show how abduction can be used to query the user for the missing examples, in order to preserve the computational advantages of extensional approaches, while guaranteeing that a correct solution be found.
Suppose that a learning system has to cover the positive example $P(a, b)$ and that the following candidate clause has been generated:

$$
P(X, Y):-\alpha(X, Y), Q(Y, Z)
$$

Moreover, we know that $\alpha(a, b)$ is true (for example because every literal in $\alpha$ is extensionally evaluated to true). Then, that clause will extensionally cover $P(a, b)$ only if there exists some value $c$ such that $Q(b, c)$ is a positive example (known to the system) of $Q$. Suppose that all such examples are missing. From the classical abductive inference rule:

$$
\alpha \leftarrow \beta \frac{\alpha}{\beta}
$$

we get:

$$
\begin{array}{lr}
P(a, b):-\exists Z \alpha(a, b), Q(b, Z) . & P(a, b) \\
\hline \exists Z Q(b, Z) &
\end{array}
$$

and then the user can be queried for a value of $Z$ such that $Q(b, Z)$ is true. The and then the set of positive examples and $P(a, b)$ can now (he result of abductive inference is not asserted as true, but only proposed as a possible truth, which is then queried to the user. It is clear that if there is no value $Z$ such that $Q(b, Z)$ is true then the clause must be discarded.

The above basic idea of generating abductive queries can be applied in a systematic way. That is, we may build chains of abductive queries, or chains of controlled abductive inference steps. A backward reasoning from an initial example that we know to be true (and that must be extensionally covered), up to what must be true in order for that initial example to be covered The chain of abductive steps can be depicted as in Figure 1.

That is, a chain is produced because the queried examples are treated in the same way as the starting one. The added examples are used to generate other abductive queries again and again until no more examples can be added, and the backward reasoning can stop. We show this on a very simple example.

Let the following hypothesis space HS be given, containing only the correct clauses for the append concept (or program):

$\{\mathrm{cl}=\operatorname{app}(\mathrm{X}, \mathrm{Y}, \mathrm{Z}):-\operatorname{head}(\mathrm{H}, \mathrm{H}), \operatorname{tail}(\mathrm{X}, \mathrm{T}), \operatorname{app}(\mathrm{T}, \mathrm{Y}, \mathrm{W}), \operatorname{cons}(\mathrm{H}, \mathrm{W}, \mathrm{Z})$.

$\mathrm{c} 2=\operatorname{app}(X, Y . Z):-\operatorname{null}(X)$, equal $(Y, Z)$.

Where all the predicates (except for $a p p$ ) are intensionally defined as usual. Wet the only given positive example be: $e_{1}=$ "app $[[a, b][c][a, b, c]) "$ A correct Let the only given positive example be: $e_{1}=$ "app [a,b] Ic], [a,b,c])" A cors program for append can oxe exterion ample. However, let us apply the abductive queries as described above. Example $e_{1}$ is matched against the head of $\mathrm{cl}$ producing the query "app([b],[c],W)?". That 


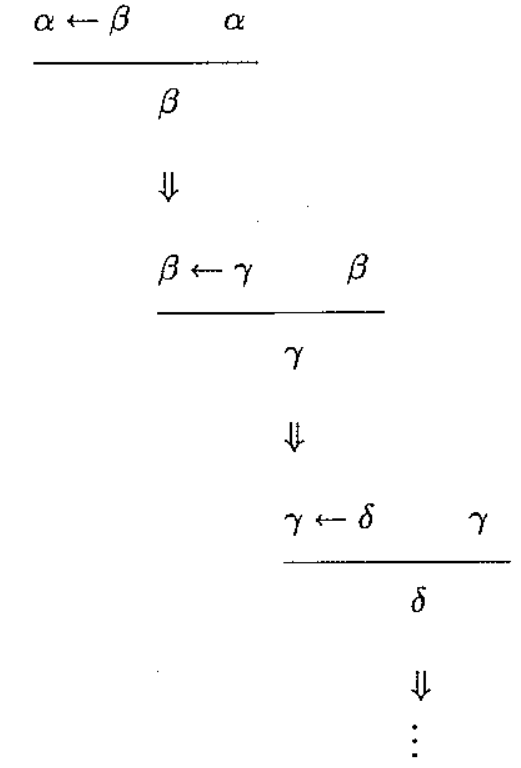

Figure 1. A chain of abductive steps

is, the user is queried for a value of the variable $W$ such that app $([b],[c], W)$ is a positive example of append. Since the answer is " $\mathrm{W}=[\mathrm{b}, \mathrm{c}]$ ", the new example $e_{2}=$ "app([b],[c],[b,c])" is added to the set of known examples of append and treated in the same way. It is matched against $\mathrm{c} 1$ producing the new example $e_{3}=$ "app([],[c],[c])". Now, $e_{3}$ is handled in the same way, but it cannot be extensionally covered by $\mathrm{cl}$, as the evaluation of "head([],H)" fails. So, it is matched with the head of clause 22 that is found to cover the example. Since no more examples are added and all the exan the abductive reasoning can stop and so also the learning task. Clauses $\mathrm{cl}$ and $\mathrm{c} 2$ have been learned.

In fact, the effect of building a chain of controlled abductive steps is that of producing a derivation tree in reverse order for the starting example $e$. That is, from $e$ to the leaves of the tree, that will be clauses from the background knowledge, known (or queried) examples, and clauses from the hypothesis space (i.e. learned known (or
clauses).

If the same is done for each positive example given initially, and recursively for each queried positive example, then the first problem of extensionality is solved. If there exists a derivation tree for a positive example in the set \{hypothesis space + background knowledge + known examples\} such a tree is found, and together with it also the clauses necessary to the derivation. The found clauses belonging to the hypothesis space will be part of the description of the target concept.
However, the second problem of extensionality still remains. How can we avoid a (known) negative example to be derived by a learned description? In fact, the same strategy can be used, but in a complementary way. For each negative exsame we test the existence of a derivation tree for it. If such a tree is found, ample, we test hie existen description of the target concept. Let us show this with an example.

Consider the following (wrong) program $\mathrm{P}$ for reverse:

$\mathrm{c} 1=\operatorname{reverse}(\mathrm{X}, \mathrm{Y}):-$ head $(\mathrm{X}, \mathrm{H}), \operatorname{tail}(\mathrm{X}, \mathrm{T} 1)$, head $(\mathrm{Y}, \mathrm{H}), \operatorname{tail}(\mathrm{Y}, \mathrm{T} 2)$, reverse(T1,T2). $\mathrm{c} 2=\operatorname{reverse}(X, Y):-\operatorname{null}(X)$, null $(Y)$.

$\mathrm{c} 3=\operatorname{reverse}([\mathrm{X}, \mathrm{Y}, \mathrm{Z}],[\mathrm{Z}, \mathrm{Y}, \mathrm{X}])$

that can be learned by using this set of examples:

$e_{1}=\operatorname{reverse}+([],[]), e_{2}=$ reverse $+([1],[1]), e_{3}=$ reverse $+([3,2,1],[1,2,3])$ $e_{4}=$ reverse $-([3,2,1],[3,2,1])$.

Then $\mathrm{P} \vdash$ reverse $([3,2,1],[3,2,1])$, however, $\mathrm{P}$ is extensionally consistent with the Then $P$ ren of a chain abductive provided examples. We car avid learing queries as follows. $e_{4}$ is matched against the head of cl gent "reverse $([2,1],[2,1])$ ?" that is classified as a negative example (let call it $e_{5}$ ) by the user. Then, $e_{5}$ is matched against $\mathrm{c} 1$ and the clause is found to extensionally cover this example, since "reverse $([1],[1])$ " is a known positive example. As a consequence, $\mathrm{c} 1$ extensionally covers a negative example (a queried one, indeed) and is rejected.

On the ground of the above examples, we have devised the following strategy. Eve constraints of the learning scheme, clause of type

$$
P(X, Y):-\alpha\left(X, Y, Z_{1}, \ldots, Z_{k}\right) .
$$

is processed with the following Abductive Completion Procedure:

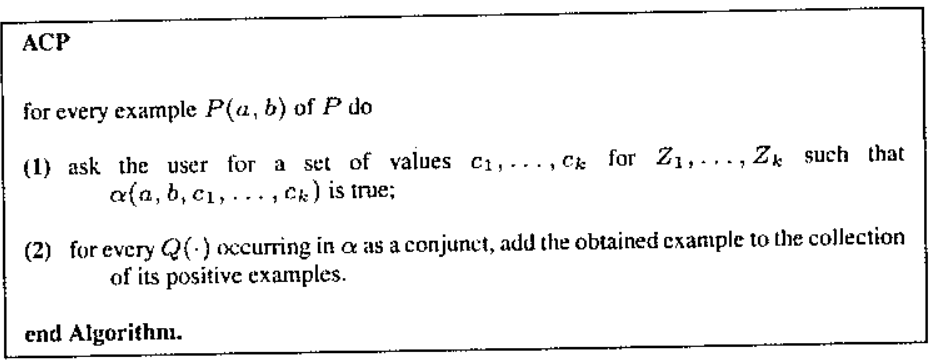

Notice that adding one example may cause the request of others. As a consequence, the procedure must be repeated for every clause, over and over, until no more examples are added.

Consider, for instance, the example about the ancestor relation given above, and the following two clauses: 
relative $(X, Y):-\operatorname{ancestor}(X, Y)$

ancestor $(X, Y) \quad:-\operatorname{parent}(W, Y)$, ancestor $(X, W)$. where

$<b, g>$ is the only positive example of "relative",

$<d, d\rangle$ is the only negative example of "relative", and

$<f, f>$ is the only negative example of "ancestor".

By using the first clause, the user is queried for ancestor $(b, g)$, and this is added to the positive examples of "ancestor". Using the second clause, the user is asked for a value of $W$ such that parent $(W, g)$ and ancestor $(b, W)$ are both true. By inspecting the Parenthood Table we have the answer $W=d$.

The second clause can be used again with $X=b$ and $Y=d$, and we obtain the completed set of positive examples for "ancestor":

$$
+\langle b, g\rangle,\langle b, d\rangle,\langle b, r\rangle
$$

Not all possible examples have been added, only the ones that were useful for those two clauses, given the initial examples. If this is done for all the clauses that are possible a priori, i.e. that satisfy the given constraints, then problems 1 and 2 no longer hold:

THEOREM 7. Suppose the examples given to an extensional learning system are completed with the ACP. If the learning system successfully exits its main loop and outputs a definition $\Pi$ (for a concept $P$, ) then $\Pi \vdash P(a, b)$ implies that $P(a, b)$ is extensionally covered by some clause in $\Pi$.

Proof. Suppose by contradiction that (c1) $\Pi \vdash P(a, b)$ but (c2) $P(a, b)$ is not extensionally covered.

Let $P(X, Y):-\alpha\left(X, Y, Z 1, \ldots, Z_{k}\right)$ be the clause resolved against $P(a, b)$ in a refutation of : $-P(a, b)$. Suppose that $P \vdash \beta(q, r) \wedge \gamma$ and $P \vdash R(r)$, but no such $r$ is a positive example of $R$. There must be one literal $R(Y)$ having this property, because of assumptions (c1) and (c2). Since the tuples of $R$ must have been completed with the given procedure, the user has been queried for $R(r)$, and this must have been inserted as a negative example. Therefore, it cannot be extensionally covered. We could now repeat the same argument for $R$. This would produce a non-terminating chain of resolution steps, and a finished proof of $Q(q)$ would never be obtained, contradicting the hypothesis that $P \vdash Q(q)$.

As an immediate corollary of the above theorem we have

COROLLARY 8. If the examples given to an extensional learning system are completed with the ACP, and the learning system successfully exits its main loop and outputs a definition $P$, then $P$ is consisten
It should be noted that this abductive completion is done as a preprocessing the The it guarantees that a solution consistent with the examples found if tep. Then, it gua consideration of previously generate clauses, as do systems (e.g. MIS [Shapiro, 1983]) that ask for new examples only when they are needed and during the learning process.

\section{DEALING WITH FUZZY RELATIONS}

It is quite interesting to notice that the relational learning framework described in the previous sections can, to some extent, be extended to the case where fuzzy relations are present. We recall that a fuzzy relation or a fuzzy predicate $P($. no longer has a binary value true or false, but can take on any value, denoted with $\tau(P(\cdot))$ from the interval $[0,1]$. Historically, the semantic of vague (or fuzzy) with $\tau(P(\cdot))$ from the interval $[0,1]$. Historically, the semantic of vague (or fuzzy)
predicates was introduced in [Zadeh, 1965]. Since it is way beyond our scope to predicates was introduced in [Zadeh, 1965]. Since it is way beyond our scope to
provide here a complete formalization of fuzzy logic, for complete details refer to now classical texts such as [Dubois and Prade, 1980; Klir and Folger, 1988; Zimmerman, 1984] and to [Léa Sombé, 1990] for an interesting and in depth discussion of formalism for uncertain reasoning, including fuzzy logic.

In order to be able to deal with fuzzy predicates or relations we need to appropriately extend all the definitions introduced in the previous sections.

\subsection{Fuzzy operators}

In order to define a form of fuzzy abduction useful for our discussions we need to recall a few definitions.

- The standard logical operators can be extended in many ways, one possibility is the following

Fuzzy Logic Operators

\begin{tabular}{cccc}
\hline$\neg P$ & $P \vee Q$ & $P \wedge Q$ & $P \rightarrow Q$ \\
$1-\tau(P)$ & $\max (\tau(P), \tau(Q))$ & $\min (\tau(P), \tau(Q))$ & $\min (\tau(Q)-\tau(P)+1,1)$
\end{tabular}

- The extension of Modus Ponens in fuzzy logic can be explained as follows

$$
\tau(Q) \geq \min (\tau(P \rightarrow Q), \tau(P))
$$

We can then propose the following fuzzy abductive inference rule

$$
\frac{\alpha \leftarrow \beta \quad \alpha}{\beta}
$$

$\tau(\beta) \leq \tau(\alpha)$ 


\subsection{Learning fuzzy relations from examples}

It is clear that when we talk about learning fuzzy relations from examples, we are not going to consider positive or negative examples, but instead examples with a"degree of positivity", i.e. the label is a number between zero and one. As a subcase, we can also have binary relations whose definitions depend upon fuzzy predicates as well.

The concept of "extensional definition" of a predicate is not affected by its fuzzyness. As in the binary case, a predicate is extensionally defined if all its values are given. For instance, the unary predicate"old $(x)$ " can be extensively defined over the universe where the parenthood table is defined. The meaning of extensional evaluation, though, needs some new formalization.

DEFINITION 9. Let $\alpha\left(X_{1}, X_{2}, \ldots, X_{n}\right)$ be a conjunction of (possibly fuzzy) predicates where there is at least one occurrence of each of the variables (not necessarily distinct) $X_{1}, \ldots, X_{n}$. Let $0 \leq \theta \leq 1$. We say that $\alpha\left(a_{1}, a_{2}, \ldots, a_{n}\right)$ is extensionally $\theta$-true if and only if

- in case $\alpha\left(X_{1}, \ldots, X_{n}\right) \equiv Q\left(X_{1}, \ldots, X_{n}\right)$, for some extensionally defined predicate $Q$, then $Q\left(a_{1}, \ldots, a_{n}\right)$ is an example of $Q$ with degree of positivity at least $\theta$.

- in case

$$
\alpha \equiv \gamma\left(X_{i_{1}}, \ldots, X_{i_{h}}, T_{1}, \ldots, T_{l}\right), Q\left(T_{1}, \ldots, T_{l}, X_{j_{1}}, \ldots, X_{j_{k}}\right),
$$

for some conjunction $\gamma$, predicate $Q, l \geq 0$ and

$$
\left\{X_{i_{1}}, \ldots, X_{i_{h}}, X_{j_{1}}, \ldots, X_{j_{k}}\right\}=\left\{X_{1}, \ldots, X_{n}\right\},
$$

then there must exist $e_{1}, \ldots, e_{l}$ such that both the conjunction $\gamma\left(a_{i_{1}}, \ldots, a_{i_{h}}\right.$ $\left.e_{1}, \ldots, e_{l}\right)$ and $Q\left(e_{1}, \ldots, e_{l}, a_{j_{1}}, \ldots, a_{j_{k}}\right)$ must be extensionally $\theta$-true.

We say that the conjunct $\alpha\left(X_{1}, \ldots, X_{n}\right)$ extensionally $\theta$-covers an example of $P$, $P\left(a_{1}, \ldots, a_{n}\right)$ if and only if $\alpha\left(a_{1}, \ldots, a_{n}\right)$ is extensionally $\theta$-true.

Given the above definition and a collection of examples for $P$ of type $\langle x, y\rangle$, $\tau(P(x, y))>$, we can act as follows:

- Sort in non-decreasing order $0 \leq \theta_{1} \leq \cdots \leq \theta_{n} \leq 1$ the values of the examples;

- for each value $\theta_{i}>0$ apply Algorithm 1 (Simplified Foil) by using the $\theta_{i}$-covering definition. Basically $\theta_{i}$ is used as a threshold value. All examples with value at least $\theta_{i}$ will be considered as "positive" and the others as negative

- if for some value $\theta_{i}$ the program terminates and outputs a logic program $\Pi_{i}$ we check whether $\Pi_{i}$ is also a"good" output for any $\theta_{j}$ with $j \neq i$.
- If so the final output will be $\Pi_{i}$ otherwise there will be no output.

Basically, we are looking for a program II such that for any example of type $(\langle x, y\rangle, \theta)$, with $\theta>0$ the following two conditions are satisfied

(C1) there exists a threshold value $\theta^{\prime} \leq \theta$ for which $\langle x, y\rangle$ is $\theta^{\prime}$-covered;

(C2) for any threshold value $\left.\theta^{\prime}\right\rangle \theta,\langle x, y\rangle$ is not $\theta^{\prime}$-covered.

Since, by fixing a threshold value, we reduce the fuzzy learning problem to the binary one we can extend definition 3 as follows

DEFINITION 10. Let $0<\theta \leq 1$ and let $E^{+, \theta}$ and $E^{-, \theta}$ be respectively, the set of examples whose value is at least $\theta$ and the set of examples whose value is less than $\theta$.

A definition $\Pi$ of $P$ is $\theta$-complete with respect to the examples $E^{+, \theta}$ of $P$ if and only if

$$
\left(\forall e \in E^{+, \theta}\right) \Pi \vdash P(e) .
$$

definition $\Pi$ of $P$ is $\theta$-consistent with respect to the examples $E^{-, \theta}$ if and only

$$
\left(\forall e \in E^{-, \theta}\right) \Pi \nvdash P(e) .
$$

Therefore, we can formalize our learning goal by saying that we are looking for There values of the given examples.

For instance, suppose we want to learn the target concept $T A$ Tall Ancestor Fo us a need to provide the truth We use again the universe of the parentho For instance suppose such values are

Tall Table

$$
\begin{array}{ll|ll}
\mathrm{a} & .3 & \mathrm{~b} & .5 \\
\mathrm{c} & .6 & \mathrm{~d} & .7 \\
\mathrm{f} & .8 & \mathrm{~g} & .9 \\
\mathrm{p} & .1 & \mathrm{r} & .6 \\
\mathrm{~s} & .6 & \mathrm{t} & .5
\end{array}
$$

Suppose also that the set of examples is the following

$>0(<r, g>, .6),(<b, g>, .5),(<b, d>, .5),(<d, g>, .7),(<b, r>$ $. .5),(<r, d>, .6),(<r, p>, .6)$;

$=0(<d, d>, 0),(<g, p>, 0)(<d, p>, 0)$. 
Then by using $\theta_{1}=.5$, Foil will output the following program

$\mathrm{TA}(X, Y):-\quad \operatorname{parent}(W, Y), \mathrm{TA}(X, W)$

$\operatorname{TA}(X, Y):-\operatorname{parent}(X, Y), \operatorname{tall}(X)$

It is easy to see that for any $\theta>0$ such a program covers all the "positive" examples and no"negative" examples. However, differences in the given data may cexamp $(<r, d>, 5)$ then the ser as example $(<r, d>, .5)$ then the second clause will $\theta$ cover it as a "positive" example for $\theta=.5$ but it will also cover it as a "negative" example for $\theta=.6$ since $\operatorname{tall}(r)=.6$ and parent $(r, d)$ has value 1 .

\subsection{Using the fuzzy abduction rule}

Obviously, the same problems that we described when dealing with binary relations are also present in the case of fuzzy relations. Moreover, we have some other problems related to the fuzzyness of some of the relations or predicates involved. Indeed, the user may have provided fuzzy truth values for such relations or predicates which are somehow inconsistent with the values attached to the examples. In this case, completing the set of examples using the proposed fuzzy abduction rule will also force the user to change if necessary the values which he/she has provided for the fuzzy predicates or relations. Indeed, suppose the system has to $\theta$-cover the example $P(x, y)=\theta$ for some $x, y$ and that the following candidate clause has been generated:

$$
P(X, Y):-\alpha(X, Y), Q(Y, Z) .
$$

Moreover, suppose that $\alpha(x, y)$ is extensionally $\theta^{\prime}$-true for every $\theta^{\prime} \geq \theta$. Then that clause

(case 1) will extensionally $\theta$-cover $P(x, y)$ if there exists some value $z$ such that $Q(y, z)$ is an example (known to the system) of $Q$ with value $\theta$; and

(case 2) it will not $\theta^{\prime}$-cover $P(x, y)$ for $\theta^{\prime}>\theta$ only if there is no value $z$ such that $Q(y, z)$ is an example (known to the system) of $Q$ with value $\theta^{\prime}>\theta$

Three cases are possible

(a) there exists one $z$ such that $Q(z, y)$ has value $\theta$

(b) there exists $w$ such that $Q(w, z)$ has value less than $\theta$ and greater than zero but there is no $z$ such that $Q(z, y)$ has value $\theta$;

(c) for any $z Q(z, y)$ has value greater than $\theta$.

If (a) holds, using our fuzzy abduction rule we can ask for an example $z$ for which $Q(z, y)$ has value $\theta$ and will add it to the list of examples. If (b) holds, the clause can never $\theta$-cover $P(x, y)$. Therefore, we would have a definition which is not $\theta$ complete. However, the example might be covered at a lower threshold and for no threshold value a "negative" example will be covered. The user must decide at this point whether there is an inconsistency in the data given to the system or whether to discard the clause

If instead (c) holds, our fuzzy abduction rule will not return any value therefore the clause will be discarded.

\section{CLASSIFICATION RULES WITH NON BINARY PREDICATES}

Abductive completion of collection of examples during a learning process can be quite useful even when dealing with non-fuzzy classification rules that contain learning classification rules in such a setting has been proposed in [Bergadano and Cuttello, 1993; Bergadano and Cutello, 1995; Bergadano and Cutello, 1995]. The main goal of this model is to introduce a theoretical framework for tuning membership functions inside a non-fuzzy classification system so to make the classification system probably, approximately correct as defined in [Valiant, 1984]. We recall that the learning process in such a case requires that the learned classification system is approximately correct with high probability and that the induction procedure is polynomial.

Under the requirement that the membership functions of the fuzzy predicates to be learned occur in a structurally given classification system, some interesting results were obtained. Though the general learning problem is computationally hard, under meaningful restrictions either on the underlying probability distribution or on the syntactic structure of the classification systems, fuzzy membership functions can be quickly learned by observing positive and negative examples. The obrined The obtained me ch a classification problem future examples will be correctly classified with high probability.

\subsection{The learning model}

Let $\mathcal{C}$ be a given concept class over a universe $\mathcal{U}$. For the time being we will Let $\mathcal{C}$ be ${ }$ of real numbers whose intended meaning is to characterize specific subparts of the system under study. Therefore, $k$ is the global dimension of the problem.

Let $C \in \mathcal{C}$ and suppose a system $S_{m}$ of classification rules for $C$ is given in the following form:

$$
\begin{array}{rll}
C(x) & :- & Q_{1}^{1}\left(p_{Q_{1}^{1}}(x)\right), \cdots, Q_{n_{1}}^{1}\left(p_{Q_{n_{1}}^{1}}(x)\right) \\
\cdots & \cdots & \cdots \\
C(x) & :- & Q_{1}^{m}\left(p_{Q_{1}^{m}}(x)\right), \cdots, Q_{n_{m}}^{m}\left(p_{Q_{n_{m}}^{m}}^{m}(x)\right)
\end{array}
$$


where

- the predicates $Q_{i}^{j}$ are taken from a set $\mathcal{P}=\left\{Q_{1}, \ldots, Q_{n}\right\}$ of given unary predicates;

- for all $Q_{j}, p_{Q_{j}}$ is a projection function that returns the parameter which is of significance for $Q_{j}$

- for every component $i$, the predicates $Q_{1}, \ldots, Q_{\pi_{i}}$ corresponding to $i$ (i.e. such that $p_{Q_{j}}$ returns the value of the $i$-th component for all $1 \leq j \leq m_{i}$ ) define a linguistic order.

Notice that

- it is possible that the same predicate occurs in different rules;

- concerning linguistic orders we have that the membership functions must be ordered in the following sense: if $j>i$ then for every $0<\alpha<1$ we have $\left\{x \mid Q_{i}(x)>\alpha\right\} \prec\left\{x \mid Q_{j}(x)>\alpha\right\}$, where $\prec$ is a suitably defined order $\left\{x \mid Q_{i}(x)\right.$
relation.

The intuitive meaning of the rule system is that a given individual $x$ is classified as a positive example for the concept $C$ if one or more of the rule antecedents are true for $x$.

Therefore we can say that $x \in \mathcal{U}$ is a member of the concept $C$ i.e. $C(x)$ is true if and only if

$$
\tau\left(\bigvee_{j=1}^{n} \bigwedge_{i=1}^{n_{j}} Q_{i}^{j}\left(p_{Q_{i}^{j}}(x)\right)\right)=1
$$

where $\tau$ is the the truth function. Since we are allowing some predicates in $\mathcal{P}$ to be fuzzy whereas $C$ is not we make the following assumptions:

- A security parameter $0<\theta<1$ is given;

- $C(x)$ is $\theta$-true if and only if

$$
\tau\left(\bigvee_{j=1}^{m} \bigwedge_{i=1}^{n_{j}} Q_{i}^{j}\left(p_{Q_{i}^{j}}(x)\right)\right)>\theta
$$

and where the truth value above is computed according to the min-max semantic, i.e.

$$
\tau\left(\bigvee_{j=1}^{m} \bigwedge_{i=1}^{n_{j}} Q_{i}^{j}\left(p_{Q_{i}^{j}}(x)\right)\right)=\max _{j=1, \ldots, m}\left(\min _{i=1, \ldots, n_{j}}\left(Q_{i}^{j}\left(p_{Q_{i}^{j}}(x)\right)\right)\right.
$$

Given a set $\mathrm{F}=\left\{\mu_{1}, \ldots, \mu_{n}\right\}$ of membership functions associated to the predicates in $\mathcal{P}$, the fuzzy classification system (FCS for short) (1) will be denoted by $S_{m}^{F}$. The notation $S_{m}$ will then denote the collection of all possible FCS's $S_{m}^{F}$, which in turn can be characterized as the collection of all sets $F$ of membership functions.

We will suppose that the unknown membership functions we are trying to learn are convex. In particular, the above implies that membership functions do not have con local maxima Moreover, colcot $Q$ the $\{x \mid Q(x)>\alpha\}$ is an interval of the real implies that for every predicate $Q$ the set $\{x \mid Q(x)>\alpha\}$ is an interval of the real line for every $\alpha$. So, we can suppose that the order relation $\prec$ is the standard orde relation on real intervals:

$$
[a, b] \prec[c, d] \text { iff } a \leq c \text { and } b \leq d .
$$

Such FCS's will be called convex fuzzy classification systems.

By definition, $S_{m}$ is connected to a non fuzzy predicate, i.e. non fuzzy conB. Our goa is to lean the memberhip functions from positive and negative cept $C$. Our goal is to lear $C$ in the hypo hesis that a classification system $S_{F}$ will examples of the concept $C$, in the hypothesis that a classifcatill be used with respect to the fixed threshold value $\theta$. The obtained truth value will be denoted by $S_{m}^{F}(x)$. In the end, $x$ is classified as a $\theta$-positive example of $C$ if $S_{m}^{F}(x)>\theta$.

\subsection{Learnability of FCS}

As proven in [Bergadano and Cuttello, 1993; Bergadano and Cutello, 1995], the problem of PAC-learning the class of convex FCS can be approached as follows:

- given $\epsilon$ and $\delta$ draw at least

$$
\max \left(\frac{4}{\epsilon} \ln \frac{2}{\delta}, \frac{16 m}{\epsilon} \ln \frac{13}{\epsilon}\right),
$$

examples from the distribution $D$;

- determine the hypercubes of $\Re^{k}$ where there are $\theta$-positive examples only (the positive hypercubes); suppose the hypercubes are $m^{\prime} \leq m$;

- use one rule of $S_{m}$ to cover each $\theta$-positive hypercube.

- project the $m^{\prime}$ positive hypercubes on the $i$-th component, obtaining $m_{i}^{\prime} \leq$ $m_{i}$ positive intervals $\left\{\left[a_{1}, b_{1}\right], \ldots,\left[a_{m_{\mathrm{i}}}, b_{m_{i}}\right]\right\}$

- define $Q_{j}^{i}$ as a trapezoidal fuzzy set using the above defined intervals.

The following result holds (see [Bergadano and Cuttello, 1993; Bergadano and Cutello, 1995]).

THEOREM 11. The general learnability problem is $\mathcal{N} \mathcal{P}$-hard. 
Similarly to any The theorem is proven by using the result proven in [Pitt and Valiant, 1988] that learning a $K$-term DNF by a $K$-term DNF is $\mathcal{N P}$-hard. We recall that a $K$-term DNF is a boolean formula of type:

$$
\bigvee_{i=1}^{K} M_{i}
$$

where the $M_{i}$ are monomials, i.e. for all $i=1,2, \ldots, K$

$$
M_{i}=x_{i_{1}} x_{i_{2}} \cdots x_{i_{a}} \cdot \bar{x}_{i_{a+1}} \cdots \bar{x}_{i_{n_{i}}}
$$

In details the problem of learning $K$-term DNF by $K$-term DNF is reduced to the problem of learning $S_{K}$ by $S_{K}$

PAC learnability results are obtained by imposing some restrictions. In particular, if we assume that the underlying distribution is such that every rule fires at least once then the learning problem can be quite easily solved. Notice in particular that this assumption is quite reasonable, as we may suppose that the classification rule is given by an expert who will not include a rule in $S_{m}$ if it is totally useless relatively to the world (i.e. the probability distribution of events) he/she lives in.

\subsection{Completing the set of examples}

All the results above cited were obtained under the following assumptions:

(a1) The teacher (i.e. the experts who provides the structure of the classification system and the labels for the examples) does not need to justify the rules in the system;

(a2) the classification system may not be minimal, i.e. there may be"redundan rules";

(a3) for each component the linguistic order is given.

In order to bypass the hardness result it is obvious that more power must be given to the learner. In particular in [Bergadano and Cutello, 1995] it was introduced the notion of "equivalence query". The learner was allowed to guess and ask for counter examples. Using this powerful tool and still under assumptions (a1)-(a3), PAC-learnability was achieved.

We now propose a way to extend the results obtained and get rid of condition (a1)-(a3). In details, the abductive completion procedure (ACP, section 6) in such framework can be adjusted as follows:

for each rule

$$
Q_{1}\left(p_{Q_{1}}(x)\right) \wedge \cdots \wedge Q_{n}\left(p_{Q_{n}}(x)\right) \rightarrow C(x)
$$

occurring in the system, we ask for a positive example $x$ such that

$$
\max _{j=1, \ldots, m}\left(\min _{i=1, \ldots, n_{j}}\left(Q_{i}^{j}\left(p_{Q_{i}^{j}}(x)\right)\right)=\min _{i=1, \ldots, n}\left(Q_{i}\left(p_{Q_{i}}(x)\right) .\right.\right.
$$

If no such a tuple $x$ exists then the rule is dropped from the classification system. It is then clear that

- by forcing the teacher to justify the given rules, the system obtained is"minimized", that is to say rules which have no influence in the classification process (no matter what the value of $\theta$ is) are eliminated.

- using a polynomially bounded number of examples, we are able in polynomial time to produce a probably approximately correct classification system, i.e. we achieve PAC-learnability;

- finally, the learner no longer needs to be given the linguistic orders for each component.

\section{AUTOMATIC ABDUCTION OF KNOWLEDGE: TESTING}

We have seen as abduction can be used in conjunction with induction, in order to alleviate the intrinsic weaknesses of this learning paradigm. However, one may reasonable wonder at which conditions such approach is practical. There are two main issues to consider. First, the abductive procedure terminates only if there is finite number of answers to each question. This is the case if we are in a finite a the approach is still acceptable if we work with functional relations. By imposin adequate input/output constraints [Bergadano and Gunetti, 1993], it is possible to work only with positive examples. For each input tuple of the target relation, there is only one positive example matching the tuple, and infinite negative examples. the system can be instructed to work only with the positive examples, assuming the negative examples being all the examples with the same input as the positive nes and different ouput values. As a consequence, for each query, the user must provide exactly one positive example, and no more.

provide exactly one positive example, and no more. Second, the number of queries to the user must not only be finite, but also quite
small, unless a database of examples is available, and the queries can be handled automatically. This depends mainly on the size of the hypothesis space, i.e. on the number of clauses that can be used to generate queries. In fact, it is becoming more and more clear as learning complex first order theories is quite difficult, and lot of knowledge must be used. As a consequence the user must not be a passive al of jow providing he examples of he taret relaton. She must also be able to agent, just providing the examples of the target relation. She must also be able to design a restricted hypothesis space on the basis of the sought relation [Bergadano and Gunetti, 1996; Van Laer et al., 1994]. Also the number of examples provided initially may influence the number of queries. Experiments based on the presented 
approach have shown as it is possible to learn complex relations by using very few representative examples of the target concept, and letting the abductive procedure asking for the missing ones. For example, it was possible to learn a program for inserting a key into a balanced tree (rebalancing the tree if necessary) by using an extensional approach and just one well chosen initial positive exan wing hypothesis space of $2^{24}$ clauses the user was quen for 15 mive the program could be learned 1831 seconds on a Sun Sparcstation 5 . The the program could be learned in 1831 seconds on a Sun Sparcstation 5. The program was composed of 9 clauses, eight of them recursive [Bergadano and Gunetti, 1996]. In general, it can be shown that an initial positive example is sufficient to learn all the clauses necessary to derive it, if the abductive completion is applied.

In this section we illustrate an interesting application of this combination of abduction and induction, outside the field of Machine Learning. We present a technique to test the correctness of a given program where, moreover, (some of) the test cases are automatically generated by the Abductive Completion Procedure through queries made to the program being tested.

The learning procedure described in Algorithm 1 outputs a set of Horn clauses. As well known, this can be regarded as a logic program. As a consequence, in the rest of this section we will use the term Inductive Program Learming (IPL) in place of concept learning, and we will speak of Logic Programs instead of concepts described through a set of Horn clauses.

\subsection{Induction and Testing}

Testing is the field of Computer Science concerned with the problem of detecting the presence of errors in programs by executing them on specific and well chosen input data. A set of test data allowing us to discover all the errors in the progren is called adequate. Though it can be for to discover all thecifor is carere an adequate set of test data for an arbitrovy program generate an adequate set of test data for an arbitrary program, testing is necessary and widely used in software development. It is practical and (relatively) easy to apply, and can give some (even very precise) information about the correctness of a program.

Testing can be seen as a way of distinguishing a program from all the possible (syntactically correct) alternatives to that program. This is very similar to IPL, where a program in the hypothesis space must be identified from among all the other possible programs on the basis of the given examples. More precisely, testing and IPL are, in some way, symmetric. The latter goes from examples to programs, whereas the former goes from programs to examples (input values).

Most of the time, a program is tested on the basis of the "correct program the user has in mind". It is common in this case to assume that although the correct program is unknown, there is a known set of programs that can be seen as alternative implementations and should contin af least one correct soluin. This concept of program correctness leads to a practical definition of meaningful test data ([DeMillo and Offutt, 1991]):
DEFINITION 12. ([Howden, 1976]) A test set $\mathrm{T}$ for a program $\mathrm{P}$ is reliable (with respect to a correct program $P_{c}$ ) iff $\left[\forall x \in T \quad P(x)=P_{c}(x)\right] \rightarrow P \equiv P_{c}$ If $P$ contains errors, this will be shown by the test cases in $\mathrm{T}$.

DEFINITION 13. Let there be given a test set $T$ for a program $P$ and a set $\mathcal{P}$ of alternative programs. We say that $\mathrm{T}$ is adequate with respect to $\mathcal{P}$ if and only if it is reliable with respect to every program in $\mathcal{P}$.

As a consequence, if $\mathcal{P}$ contains at least one correct program $P_{c}$, then the test set will also be reliable for $P$. Many different testing techniques are based on a relation between the program $P$ to be tested and a set of possible altematives $P$ In particular, fault-based methods [DeMillo and Offutt, 1991; King and Offutt, 1991] use a set of typical programming errors defined a priori, and alternative
programs are obtained by inserting some of these errors (that is, possible program mutations) in $P$.

Testing based on the inductive learning of logic programs also relies on definition 13 of adequateness of a test set. As noted above, an intuitive symmetry between induction and testing can almost immedintly be noticed [Weyuker, 1983] programs to input values. Given a test set $T$ of input values for a program $P$, the examples of $P$ for $T$ are defined as $E(P, T)=\{<i, o>\mid i \in T$ and $P(i)=0\}$. This notion is formalized by Weyuker as follows [Weyuker, 1983]:

DEFINITION 14. A test set $\mathrm{T}$ is program adequate for a program $\mathrm{P}$ if and only if $P_{I}$ is inductively inferred from $\mathrm{E}(\mathrm{P}, \mathrm{T})$ and $P_{I} \equiv \mathrm{P}$.

The intuitive meaning of the above definition is as follows: If, given a set $E(P, T)$ of input/output examples, we inductively infer a program $P_{I} \equiv P$, then $T$ is likely to be useful for testing the program $P$.

In the previous sections we have described a a basic learning method which is terminating and explicitly make use of a finite set of legal programs. More precisely, the hypothesis space is made of Horn clauses, and every possible subset of such Horn clauses is a program that could be learned by the induction procedure, on the basis of the given examples. We will call $\mathcal{P}$ the set of all possible programs. If there are $n$ Horn clauses, there are $2^{n}$ possible legal programs in $\mathcal{P}$.

The learned program $P_{I}$ must belong to $\mathcal{P}$. This is consistent with the fact that all the test case generation procedures that are not specification based refer (sometimes implicitly) to a set $\mathcal{P}$ of alternative programs. As a consequence, definition 14 can then be stated in terms of "sound and complete IPL": T is adequate for P if and only if $P$ is the only program that can be learned from $E(T, P)$ with a sound and costriction to a finite set of pothesis space) $\mathcal{P}$ seems to be acceptable for testing; for instance, all approaches

${ }^{2}$ Formally, a system is defined to be sound if it only outputs programs that are consistent w.r.t. the in the given hypothesis space. As an instance, the leaming procedure of Algorithm 1 (Simplified Foil). is neither sound nor complete, by virtue of Problems 1 and 2 . 
to fault-based testing such as mutation analysis, are based on this assumption.

The intuitive symmetry between induction and testing can now be made clear: induction is an inference from the pair $\angle E(T, P), \mathcal{P}>$ to a program $P$ in $\mathcal{P}$, whereas testing is an inference from $\angle P, P>$ to the test $>t T$. In the next subection this symmetry will be used in actually generating the adequate test cases for a (not necessarily logic) program.

\subsection{Abductive Test Case Generation}

Let us call SFOIL (Simplified Foil) the learning procedure of Algorithm 1. Let us call $S F O I L_{A C P}$ the SFOIL algorithm augmented with the Abductive Completion Procedure and with input/output constraints, as discussed at the beginning of this section. A sequence of inductions of programs from examples is used to generate test cases. Initially, there are no examples and, in the end, the generated set of examples will be adequate in the sense of definition 13 .

Let $\mathrm{P}$ be the program to be tested. At any given moment, the examples generated so far are used to induce a program $\mathrm{P}^{\prime}$. New examples that distinguish $\mathrm{P}$ from $\mathrm{P}^{\prime}$ are added, and the process is repeated until no program $P^{\prime}$ that is not equivalent to $\mathrm{P}$ can be generated. This procedure is described in more detail below.

Test case generation procedure:

Input: a program $P$ to be tested,

$$
\text { a finite set } \mathcal{P} \text { of alternative programs }
$$

Output: an adequate test set $T$

$\mathrm{T} \leftarrow \emptyset$

loop:

$\left\langle\mathrm{P}^{\prime}, \mathrm{T}\right\rangle \leftarrow S F O I L_{A C P}(\mathrm{E}(\mathrm{T}, \mathrm{P}), \mathcal{P})$

if $\mathrm{P}^{\prime}=$ " $\mathrm{Fail}$ " then return $\mathrm{T}$

if $(\exists i) P^{\prime}(i) \neq P(i)$

then $\mathrm{T} \leftarrow \mathrm{T} \cup\{\mathrm{i}\}$

else $\mathcal{P} \leftarrow \mathcal{P}-\mathrm{P}^{\prime}$

goto loop

In order for the above procedure to work, the leaming algorithm $S F O I L_{A C P}$ must be sound and complete, as it is proved by theorem 7 and corollary 8 . In the test case generation procedure, the test set $\mathrm{T}$ is initially empty. The main step in the loop consists of using $S F O I L_{A C P}$ to learn a program $\mathrm{P}^{\prime}$ that is consistent with the examples generated so far. $P^{\prime}$ is then muled out either by (1) adding an input vain. When $S F O I L$ again. When $S F O I L_{A C P}$ cannot find a program $\mathrm{P}^{\prime} \in \mathcal{P}$ that is consistent with the examples, then the only programs with this property are $\mathrm{P}$ and those equivalent to it, i.e., the test set $T$ is adequate. This is proved by the following:
THEOREM 15. Let equivalence be decidable for programs in $\mathcal{P}$. Then the above test case generation procedure outputs an adequate test set $T$ for $P$.

Proof. Since equivalence is decidable for programs in $\mathcal{P}$, the procedure terminates, as the condition $(\exists i) P^{\prime}(i) \neq P(i)$ always produces an answer. Suppose, by contradiction, that the obtained test set $T$ is not adequate. This means that there is $P^{\prime} \in$ $\mathcal{P}$ and an input $\mathrm{i} \notin \mathrm{T}$ such that $\mathrm{P}^{\prime}(\mathrm{i}) \neq \mathrm{P}(\mathrm{i})$, but $(\forall \mathrm{v}) \in \mathrm{T}, \mathrm{P}^{\prime}(\mathrm{v})=\mathrm{P}(\mathrm{v})$. However, just before termination, $S F O I L_{A C P}$ failed to induce a program $\mathrm{P}_{1}$. Moreover, $\mathrm{P}^{\prime}$ is complete and consistent with respect to $E(T, P)$, because $(\forall v) \in T, P^{\prime}(v)=P(v)$. Then, just before termination, and because $S F O L_{A C P}$ is sound and complete, it $\mathcal{P}$ at some previous iteration. But, in that case, there must have been an input value i such that $\mathrm{P}^{\prime}(\mathrm{i}) \neq \mathrm{P}(\mathrm{i})$, and this value would have been added to $\mathrm{T}$. This contradicts the assumption that $\mathrm{i} \notin \mathrm{T}$ and $\mathrm{T}$ is not adequate.

If a decision procedure is available for finding an input $\mathrm{i}$ such that $\mathrm{P}(\mathrm{i}) \neq \mathrm{P}^{\prime}(\mathrm{i})$, this is used directly in the above test generation method. As this is not true in general, examples are found by enumerating (in some random or ad hoc order) the possible inputs $i$, and stopping when an $i$ is found such that $P(i)=0 \neq P^{\prime}(i)=0^{\prime}$. This enumeration could also be guided by other test case generation techniques, such as path or functional testing. The requirement of decidable equivalence is of course not easily verified or accepted. is a major theoretical and practical issue in program testing. In the implementation of our test case generation method, we approximate it by means of its time-bounded semi-decision procedure. Except for this approximation, the system produces adequate test sets with respect to any finite class of programs $\mathcal{P}$. The reader may find all the details of this testing technique in [Bergadano and Gunetti, 1996b].

\subsection{An example: testing a merge program}

Consider the problem of merging two ordered lists, where we allow them to contain repeated elements. The output list must be ordered and should contain every element of the input lists only once. This problem requires a procedure for removing the elements that are repeated. Suppose we are given the following:

remove $(X,[X \mid Y], Z):-~ !, \operatorname{remove}(X, Y, Z)$. remove(,,Y,Y).

This does just as much as necessary: it removes the initial occurrences of an element in a list, e.g., remove $(a,[a, a, b],[b])$, but remove $(a,[b, a],[b, a])$. Let the program to be tested be the following:

P:

1) $\operatorname{merge}(X, Y, Z)$ :- null $(X), Y=Z$ 
2) $\operatorname{merge}(X, Y, Z)$ :- $\operatorname{null}(Y), X=Z$.

3) merge $(X, Y, Z)$ :- head $(X, X 1)$, tail $(X, X 2)$, head $(Y, X 1)$, tail $(Y, Y 2)$, merge $(X 2, Y 2, W)$, remove $(X 1, W, T)$, cons $(X 1, T, Z)$.

4) merge $(X, Y, Z)$ :- head $(X, X 1)$, tail $(X, X 2)$, head( $Y, Y 1)$, tail $(Y, Y 2)$,

$\mathrm{X} 1<\mathrm{Y} 1$, merge $(\mathrm{X} 2, \mathrm{Y}, \mathrm{W})$, remove $(\mathrm{X} 1, \mathrm{~W}, \mathrm{~T})$, cons $(\mathrm{X} 1, \mathrm{~T}, \mathrm{Z})$

5) merge $(X, Y, Z)$ :- head $(X, X 1)$, tail $(X, X 2)$, head $(Y, Y 1)$, tail $(Y, Y 2)$,

$\mathrm{X} 1>\mathrm{Y} 1, \operatorname{merge}(\mathrm{Y} 2, \mathrm{X}, \mathrm{W})$, remove $(\mathrm{Y} 1, \mathrm{~W}, \mathrm{~T})$, cons $(\mathrm{Y} 1, \mathrm{~T}, \mathrm{Z})$.

In this program the first two clauses are wrong. In fact, any repeated element in the nonempty list will be present in the output. Let $\mathcal{P}$ be defined as any program made of any clause that can be built by using as head merge $(X, Y, Z)$ and whose body is made of a subset of the literals occurring in $P$. As a consequence, $\mathcal{P}$ contains $2^{2^{19}}$ alternative programs, i.e., all possible subsets of the space of clauses. Among the subsets there are versions of the correct implementation of merge. A concise representation of $\mathcal{P}$ will be given as input to $S F O I L_{A C P}$. Of course, $\mathcal{P}$ is not generated explicitly. See [Bergadano and Gunetti, 1996b] for the implementative details.

The test case generation procedure starts with an empty test set $T_{0}$ of input values, and calls $S F O L_{A C P}$. As $\mathrm{E}\left(\mathrm{T}_{0}, \mathrm{P}\right)$ contains no examples, the empty program $\mathrm{P}_{0}$ is an acceptable output of $\operatorname{SFOIL}_{A C P}\left(\mathrm{E}\left(\mathrm{T}_{0}, \mathrm{P}\right), \mathcal{P}\right)$

Pairs of lists $\mathrm{X}$ and $\mathrm{Y}$ are then enumerated, so that

$P_{0} \vdash$ merge $\left(X, Y, Z^{\prime}\right), P \vdash$ merge $(X, Y, Z)$ and $Z \neq Z^{\prime}$

The first such pair that is found is $\langle X, Y\rangle=\langle[],[]\rangle$; for this input, $P_{0}$ produces no output and $\mathrm{P}$ outputs $\mathrm{Z}=[\mathrm{[}]$. The new test set is then $\mathrm{T}_{1}=\{\langle[],[]\rangle\}$

$S F O I L_{A C P}\left(\mathrm{E}\left(\mathrm{T}_{1}, \mathrm{P}\right), \mathcal{P}\right)$ is called again, yielding $\mathrm{P}_{1}$ :

merge $(X, Y, Z):-Y=Z$.

This program is an acceptable output of $S F O I L_{A C P}$ because merge([],[],[]) is derived from it, and the output is the same as that of $P$.

Pairs of lists $\mathrm{X}$ and $\mathrm{Y}$ are enumerated, so that

$\mathrm{P}_{\mathrm{i}} \vdash \operatorname{merge}\left(\mathrm{X}, \mathrm{Y}, \mathrm{Z}^{\prime}\right), \mathrm{P} \vdash \operatorname{merge}(\mathrm{X}, \mathrm{Y}, \mathrm{Z})$ and $\mathrm{Z} \neq \mathrm{Z}^{\prime}$. The first such pair that is found is:

$\langle\mathrm{X}, \mathrm{Y}\rangle=\left\langle[1],[1]>\right.$; for this input, $\mathrm{P}_{1}$ outputs $\mathrm{Z}=[]$ while $\mathrm{P}$ outputs $\mathrm{Z}=[1]$. The new test set is then $\mathrm{T}_{2}=\mathrm{T}_{1} \cup\{<[1],[]>\}$.

$\operatorname{SFOIL}_{A C P}\left(\mathrm{E}\left(\mathrm{T}_{2}, \mathrm{P}\right), \mathcal{P}\right)$ is called again, yielding $\mathrm{P}_{2}$

merge $(X, Y, Z):-X=Z$.

This program is an acceptable output of $S F O I L_{A C P}$ because merge([],[],[]) and merge([1],[],[1]) are derived from it.

The test case generation goes on in this way generating new test cases and learning new programs, until no new program can be discovered and $S F O I L_{A C P}$ fails, ending the test generation process. The complete set of learned test cases, with the corresponding outputs, is the following:

1: merge([],[],[])

2: merge $([1],[],[1])$

3: merge([2],[1],[1,2])

4: merge([2],[],[2]) - abduced by $S F O I L_{A C P}$ from $P$

5: merge $\left([1,[1],[1])\right.$ - abduced by $S F O I L_{A C P}$ from $\mathrm{P}$

6: merge $([1],[1],[1])$

7: merge $([1,1,2],[1],[1,2])$

8: merge $([1,1,2],[],[1,1,2])$ - abduced by $S F O I L_{A C P}$ from $\mathrm{P}$, it shows the error

9: $\operatorname{merge}([1],[2],[1,2])$

Only nine examples have been required to locate the error, whereas many more would have been necessary in random testing, if there are many possible element values with respect to the average list length.

We see that some of the examples (one of them showing the error) are added as a consequence of the ACP. Consider, e.g., example 7. When $S F O I L_{A C P}$ is learning a program $P_{5}$ consistent with examples 1-7, it has to find a clause covering example 7. During its construction, it comes to the (partial) clause:

merge $(X, Y, Z):-\operatorname{head}(X, X 1)$, tail $(X, X 2)$, head $(Y, X 1)$, tail( $Y, Y 2)$, merge $(X, Y 2, W)$

There is no example discovered so far covering literal merge([1,1,2],[],W) and, as consequence, the ACP queries the program $P$ (that is, it runs it) for a value o such that merge $([1,1,2],[], 0)$ is true, getting the answer $0=[1,1,2]$ and producing the new testing case represented by example 8 above.

It should also be noted that $\mathrm{P}$ does not belong to the hypothesis space $\mathcal{P}$; actually this is not required by the test case generation system. In fact, the program to be tested could be used as a black box and could, in principle, be written in any programming language.

\section{CONCLUSION}

The relation between induction and abduction is briefly discussed in this paper. Wentive reasoning in $\mathrm{Ma}$ chine Learning. On one hand, it has been used to guide search in a top-down specialization framework related to Explanation-based Learning. On the other hand, we have also shown how abduction can be used to query the user for examples that may be missing. This means that the user must not provide all the needed examples to learn one definition at a time. He/she can forget some examples, and the abductive procedure will ask for them. Observe that only the 
useless examples. In many extensional systems [Muggleton and Feng, 1990; Quinlan, 1990] the user must provide all the examples at one time, and usually a superset of the examples needed is given, resulting in a lot of time wasted in covering useless examples. We have also highlighted a possible extension of such a learning framework to the case where fuzzy predicates occur. In such a case, the user not only must provide all the missing example but must also provide consistent fuzzy values for all the predicates involved.

\section{ACKNOWLEDGEMENTS}

The authors thank esprit project DRUMS II which supported part of this research.

F. Bergadano and D. Gunetti

Computer Science Department, University of Torino, Italy.

V. Cutello,

Mathematics Deptartment, University of Catania, Italy.

REFERENCES

[Bergadano and Besnark, 1994] F. Bergadano and P. Besnard. Abduction and induction by nonmonotonic logics, Workshop on Mathematical and Statistical Methods in Artificial Intelligence,

Udine, Italy, 1994.
[Bergadano and Cuttello, 1993] F. Bergadano and V. Cutello. Learning membership functions. Pro. ceedings of ECSQARU' 93,2 2nd European Conference on Symbolic and Quantitative Approaches Verlag, Vol. 747, pp. 25-32,1993.

(Bergadarno and Cutello, 1995] F. Bergadano and V. Cutello. Learning fuzzy sets. In Proceedings of EUFIT'95, G. J. Zimmermann, ed. Aachen, Germany, 1995.

[Bergadano and Cutello, 1995] F. Bergadano and V. Cutello. Probably approximate correct (PAC) learning in fuzzy classification systems. IEEE Transactions on Fuzzy Systems , 3, 473-478, 1995. concept induction. Proceedings of the Fifh International . A knowledge intensive approach to 305-317, Ann Arbor, MI, 1988.
. Fifh International Conference on Machine Learning. p [Bergadano et al., 1989] F. Bergadano, A. Giordana and S. Ponsero. Deduction in Top-down Inductive
Learning, Proceedings of the Sixth International Conference on Machine Learning. pp. 23-25,

Ithaca, NY, 1989.
[Bergadano and Gunetti, 1993] F. Bergadano and D. Gunetti. An interactive system to learn functional logic programs. In Proceedings of the International Joint Conference on Artificial Intelligence,

[Bergadano and Gunetti, 1996] F. Bergadano and D. Gunetti. Inductive Logic Programming: from Mearning to Software Engineering. MIT Press, Cambridge, MA, 1996.

Bergadano and Gunetti, 1996b] F. Bergadano and D. Gunetti. Testing by means of inductive progra learning. ACM Transactions on Software Engineering and Methodology (ACM TOSEM), 5, 1996. (Bimbaum and Collins, 1991J L. Birnbaum and G. Collins, eds. Proceedings of the International $M L$ Conference, part VI: Learning Relations. Morgan Kaufmann, 1991.

Cohen, 1992] W. Cohen. Abductive explanation-based learning: a solution to the multiple inconsis-

Cohen, 1994] W. Cohen. Incremental abductive explanation-based leaming. Machine Learning, 15
[Console and Saitta, 1993] L. Console and L. Saitta. Generalization in learning and abduction. tech-

nical report, University of Torino, 1994.
[DeMillo and Offutt, 1991$]$ R. A. DeMillo and A. J. Offutt. Constraint-based automatic test data generation. IEEE Trans. on Software Engineering, 17, 900-910, 1991.

DeRaedt and Bruynooghc, 1991] L. DeRaedt and M. Bruynooghe. CLINT: A Multistrategy Interactive Concept-Learner and Theory Revision System. In R. S. Michalski and G. Tecuci, eds. Pro ceedings of the Workshop on Multhsrategy Learning, pp. 175-190, Happers Fery, WA, 1991. Ellman, 1989] T. Ellman. Exp

Computing Surveys, 21, pp. 163-222, 1989

[Howden, 1976] W. E. Howden. Reliability of the path analysis testing strategy. IEEE Trans. on Software Engineering, 2, 208-215, 1976

. Folger. Fuzzy Sets uncertainty and Information. Prentice

KKing and Offutt, 1991] K. N. King and A. J. Offutt. A Fortran language system for mutation-based software testing. Software Practice and Experience, 21, 685-718, 1991

[Léa Sombé, 1990] Léa Sombé Reasoning Under Incomplete Information in Artificial Intelligence. John Wiley and Sons Inc., 1990.

IVan Laer et al., 1994] W. Van Laer, L. Dehaspe and L. DeRaedt. Applications of a logical discovery Minge. Les 1986] T. Mitchell R. M. Keller and S. Kedar-Cabelli. Explanaven. Muggleton and Feng, 1990] S. Muggleton a Proceedings of the First Conference on Algorithmic Learning Theory, Tokyo, Japan, 1990.

[Pazzani et al. 1991] M. Pazzani, C. A. Brunk and G. Silverstein. A Knowledge-intensive approach to learning relational concepts. Proceedings of the Bit International Conference on Machne Learning
1991 .

[Pazzani and Kibler, 1992] M. Pazzan
Machine Learning, 9, 57-94, 1992.

[Pitt and Valiant, 1988] L. Pitt and L. G. Valiant. Computational limitations on learning from examples. Journal of ACM, 35, 965-984, 1988 .

Quinlan, 1990] R. Quinlan. Learning Logical Definitions from Relations. Machine Learning, 5 , [Rouveirol, 1992]

Learning. ECCAI, Vienna, Austria, 1992.
Roce

Valiant, 1984] L. Valiant. A theory of the learnable. Communications of the ACM, 27, 1134-1142,

1984.
[Weyuker, 1983] E. J. Weyuker. Assessing Test Data Adequacy Through Program Inference. ACM [Zadeh, 1965] L. A. Zadeh. Fuzzy sets. Information and Control, 8, 338-353, 1965

[Zimmerman, 1984] H. J. Zimmermann Fuzzy Set Theory and its applications. Kluwer-Nijhoff, Boston, 1984 